Bull. Korean Math. Soc. 52 (2015), No. 2, pp. 467-481

http://dx.doi.org/10.4134/BKMS.2015.52.2.467

\title{
SIGNED A-POLYNOMIALS OF GRAPHS AND POINCARÉ POLYNOMIALS OF REAL TORIC MANIFOLDS
}

\author{
Seunghyun Seo and Heesung Shin
}

\begin{abstract}
Choi and Park introduced an invariant of a finite simple graph, called signed a-number, arising from computing certain topological invariants of some specific kinds of real toric manifolds. They also found the signed a-numbers of path graphs, cycle graphs, complete graphs, and star graphs.

We introduce a signed a-polynomial which is a generalization of the signed a-number and gives $a-, b$-, and c-numbers. The signed a-polynomial of a graph $G$ is related to the Poincaré polynomial $P_{M(G)}(z)$, which is the generating function for the Betti numbers of the real toric manifold $M(G)$. We give the generating functions for the signed apolynomials of not only path graphs, cycle graphs, complete graphs, and star graphs, but also complete bipartite graphs and complete multipartite graphs. As a consequence, we find the Euler characteristic number and the Betti numbers of the real toric manifold $M(G)$ for complete multipartite graphs $G$.
\end{abstract}

\section{Introduction}

A signed a-number of a finite simple graph $G$ is a graph invariant introduced by Choi and Park [3], denote by $\operatorname{sa}(G)$, as follows:

- $\operatorname{sa}(\emptyset)=1$.

- $\mathrm{sa}(G)$ is the product of signed a-numbers of connected components of $G$.

- $\operatorname{sa}(G)=0$ if $G$ is a connected graph on odd number of vertices.

- If $G$ is connected with even number of vertices, then $\operatorname{sa}(G)$ is given by the negative of the sum of signed a-numbers of all induced subgraphs $G^{\prime}$ of $G$ except itself $G$.

Let the a-number a $(G)$ be the absolute value of the signed a-number of $G$, the $b$-number $\mathrm{b}(G)$ the sum of signed a-numbers induced subgraphs of $G$, and the $c$-numbers $\mathrm{c}_{i}(G)$ the sum of a-numbers of induced subgraphs of $G$ with $i$ vertices.

Received November 24, 2013

2010 Mathematics Subject Classification. 05A15, 05C30, 37F20.

Key words and phrases. graph invariant, toric topology, Poincaré polynomial. 
These numbers arise from computing certain topological invariants of some specific kinds of real toric manifolds which are important objects in toric topology. For a finite simple graph $G$, a building set $B(G)$ is consisting of connected induced subgraphs of $G$ and a nestohedron $P_{\mathcal{B}(G)}$ is defined as the Minkowski sum of simplices

$$
P_{\mathcal{B}(G)}=\sum_{I \in B(G)} \Delta_{I}
$$

which is called a graph associahedron. Since every nestohedron is a Delzant polytope [5, Proposition 7.10], the real toric manifold $M(G)$ can be defined as the set of real points in the toric manifold, which is associated to the normal fan of the graph associahedron $P_{\mathcal{B}(G)}$ as Delzant polytope. For further information, see $[2,5,6]$.

Recently, Choi and Park [3, Theorem 1.1] showed that the Euler characteristic $\chi(M(G))$ of $M(G)$ is equal to $\mathrm{b}(G)$ and the $i$-th rational Betti number $\beta_{i}(M(G))$ of $M(G)$ is equal to $\mathrm{c}_{2 i}(G)$. We remark that $\mathrm{c}_{2 i}(G)$ is the same with $\mathrm{a}_{i}(G)$ in [3]. They also computed these numbers of path graphs $P_{2 n}$, cycle graphs $C_{2 n}$, complete graphs $K_{2 n}$, and star graphs $K_{1,2 n-1}$.

In this paper, we introduce a signed a-polynomial which is a generalization of the signed a-number and gives a-, b-, and c-numbers. The signed a-polynomial of a graph $G$ is related to the Poincaré polynomial $P_{M(G)}(z)$, which is the generating function for the Betti numbers of the real toric manifold $M(G)$. The relation will be shown in equation (7). We give the signed a-polynomials of not only path graphs, cycle graphs, complete graphs, and star graphs, but also complete bipartite graphs $K_{p, q}$ and complete multipartite graphs $K_{p_{1}, \ldots, p_{m}}$. As a consequence, we find $\chi(M(G))$ and $\beta_{i}(M(G))$ for $G=K_{p, q}$ and $G=$ $K_{p_{1}, \ldots, p_{m}}$.

\section{Preliminaries}

From now on, we assume that a graph is finite, undirected, and simple. We rewrite a formal definition of a signed a-number $\operatorname{sa}(G)$ of a graph $G=(V, E)$ in the previous section as

$$
\operatorname{sa}(G)= \begin{cases}1 & \text { if } G \text { is the empty graph, } \\ 0 & \text { if } G \text { is connected and }|V| \text { is odd, } \\ -\sum_{V^{\prime} \subseteq V} \operatorname{sa}\left(\left.G\right|_{V^{\prime}}\right) & \text { if } G \text { is connected and }|V| \text { is even } \geq 2, \\ \prod_{G^{\prime} \in \operatorname{comp}(G)} \operatorname{sa}\left(G^{\prime}\right) & \text { if } G \text { is disconnected, }\end{cases}
$$

where $\left.G\right|_{V^{\prime}}$ is the induced subgraph of $G$ by a vertex subset $V^{\prime}$ and $\operatorname{comp}(G)$ is the set of connected components of $G$. From the above definition, it is easy to check that $\operatorname{sa}(G)=0$ for every graph $G$ with at least one connected component on odd number of vertices; and $\sum_{V^{\prime} \subseteq V} \mathrm{sa}\left(\left.G\right|_{V^{\prime}}\right)=0$ for every nonempty graph 
$G$ on $V$ with every connected component on even number of vertices. Thus, we find a simpler equivalent definition of a signed a-number as follows.

Definition 1. A signed a-number $\operatorname{sa}(G)$ of a graph $G=(V, E)$ is defined by (1)

$$
\mathrm{sa}(G)= \begin{cases}1 & \text { if } G \text { is the empty graph, } \\ 0 & \text { if } G \text { has a connected component on odd number } \\ & \text { of vertices, } \\ -\sum_{V^{\prime} \subsetneq V} \operatorname{sa}\left(\left.G\right|_{V^{\prime}}\right) & \text { otherwise. }\end{cases}
$$

Consequently, we define a-, b-, and c-numbers of a graph with the signed a-numbers.

Definition 2. The $a$-, $b$-, and $c$-numbers of a graph $G$, denoted by a $(G), \mathrm{b}(G)$, and $\mathrm{c}_{i}(G)$, are defined by

$$
\begin{aligned}
\mathrm{a}(G) & =(-1)^{|V| / 2} \mathrm{sa}(G), \\
\mathrm{b}(G) & =\sum_{V^{\prime} \subseteq V} \mathrm{sa}\left(\left.G\right|_{V^{\prime}}\right), \\
\mathrm{c}_{i}(G) & =\sum_{\substack{V^{\prime} \subseteq V \\
\left|V^{\prime}\right|=i}} \mathrm{a}\left(\left.G\right|_{V^{\prime}}\right)=(-1)^{i / 2} \sum_{\substack{V^{\prime} \subset V \\
\left|V^{\prime}\right|=i}} \mathrm{sa}\left(\left.G\right|_{V^{\prime}}\right) .
\end{aligned}
$$

By definition, for any graph $G$, it holds that $\mathrm{c}_{i}(G)=0$ if $i$ is odd, and $\mathrm{c}_{n}(G)=\mathrm{a}(G)$ if $n$ is the number of vertices of $G$. From a topological viewpoint $\left[3\right.$, Remark 2.2], it is obvious that $\mathrm{a}(G)$ and $\mathrm{c}_{i}(G)$ are nonnegative integers.

\section{On signed a-polynomials}

Now, we introduce a generalization of a-, b-, and c-numbers of graphs.

Definition 3 (Signed a-polynomial). The signed a-polynomial $\mathrm{sa}(G ; t)$ of a graph $G$ is defined by

$$
\mathrm{sa}(G ; t)=\sum_{V^{\prime} \subseteq V(G)} \mathrm{sa}\left(\left.G\right|_{V^{\prime}}\right) t^{\left|V \backslash V^{\prime}\right|},
$$

where $V(G)$ is the set of vertices of $G$.

From the equations (1)-(5), for $|V(G)|=n$, it holds that

$$
\begin{aligned}
\mathrm{sa}(G) & =\mathrm{sa}(G ; 0), & \mathrm{a}(G) & =(-1)^{n / 2} \mathrm{sa}(G ; 0), \\
\mathrm{b}(G) & =\mathrm{sa}(G ; 1), & \mathrm{c}_{i}(G) & =(-1)^{i / 2}\left[t^{n-i}\right] \mathrm{sa}(G ; t) .
\end{aligned}
$$

Thus, $\operatorname{sa}(G ; t)$ is represented as the sum of $\mathrm{c}_{i}(G)$ 's by

$$
\mathrm{sa}(G ; t)=\sum_{j=0}^{\lfloor n / 2\rfloor}(-1)^{j} c_{2 j}(G) t^{n-2 j} .
$$


For example, if $G$ is a graph obtained by deleting one edge from the complete graph $K_{4}$, then

$$
\mathrm{sa}(G ; t)=t^{4}-5 t^{2}+4 .
$$

Thus, $\mathrm{sa}(G)=\mathrm{a}(G)=4, \mathrm{~b}(G)=0$, and $\left\{\mathrm{c}_{i}(G)\right\}_{i=0}^{4}=1,0,5,0,4$.

Remark. The Poincaré polynomial $P_{M(G)}(z)=\sum_{i \geq 0} \beta_{i}(M(G)) z^{i}$ is the generating function for the Betti numbers $\beta_{i}(M(G))$ of the real toric manifold $M(G)$. Since $\beta_{i}(M(G))=\mathrm{c}_{2 i}(G)$ in $[3$, Theorem 1.1], it holds that

$$
P_{M(G)}(z)=(\sqrt{-z})^{|V|} \mathrm{sa}\left(G ; \frac{1}{\sqrt{-z}}\right) \text {. }
$$

In the rest of the section, we compute the generating functions for signed a-polynomials of path graphs, cycle graphs, complete graphs, and star graphs.

Theorem 1. Let $P_{n}$ be the path graph with $n$ vertices, which is a tree with exactly $n-2$ vertices of degree 2. Then the generating function for signed a-polynomials of $P_{n}$ is given by

$$
\sum_{n \geq 0} \operatorname{sa}\left(P_{n} ; t\right) x^{n}=\frac{-1+2 t x+\sqrt{1+4 x^{2}}}{2 t x-2\left(t^{2}-1\right) x^{2}} .
$$

Proof. From Theorem 2.5 in [3], it is known that

$$
\mathrm{c}_{2 i}\left(P_{n}\right)=\left(\begin{array}{c}
n \\
i
\end{array}\right)-\left(\begin{array}{c}
n \\
i-1
\end{array}\right)=\mathrm{Cat}_{n-i, i}
$$

with Catalan triangle numbers Cat $_{n, k}=\left(\begin{array}{c}n+k \\ k\end{array}\right)-\left(\begin{array}{c}n+k \\ k-1\end{array}\right)$. Using formula (6), we have

$$
\mathrm{sa}\left(P_{n} ; t\right)=\sum_{j=0}^{\lfloor n / 2\rfloor}(-1)^{j} \mathrm{Cat}_{n-j, j} t^{n-2 j} .
$$

Thus, we obtain

$$
\begin{aligned}
\sum_{n \geq 0} \operatorname{sa}\left(P_{n} ; t\right) x^{n} & =\sum_{n \geq 0} \sum_{j=0}^{\lfloor n / 2\rfloor}(-1)^{j} \operatorname{Cat}_{n-j, j} t^{n-2 j} x^{n} \\
& =\sum_{k \geq 0} \sum_{j \geq 0} \operatorname{Cat}_{k, j}(-x / t)^{j}(t x)^{k} .
\end{aligned}
$$

Since the generating function for Catalan triangle numbers is

$$
\sum_{n \geq 0} \sum_{i \geq 0} \operatorname{Cat}_{n, i} w^{i} z^{n}=\frac{\operatorname{Cat}(w z)}{1-z \operatorname{Cat}(w z)},
$$

where Cat $(x)=\frac{1-\sqrt{1-4 x}}{2 x}$, therefore (9) becomes (8). 
TABLE 1. Numbers for path graphs $P_{n}$, where Catalan triangle numbers Cat $_{n, k}=\left(\begin{array}{c}n+k \\ k\end{array}\right)-\left(\begin{array}{c}n+k \\ k-1\end{array}\right)$ and Catalan numbers $\mathrm{Cat}_{n}=\mathrm{Cat}_{n, n}=\frac{1}{n+1}\left(\begin{array}{c}2 n \\ n\end{array}\right)$.

\begin{tabular}{c||c|c|c}
$G$ & $P_{0}$ & $P_{2 n}$ & $P_{2 n+1}$ \\
\hline $\mathrm{sa}(G)$ & 1 & $(-1)^{n} \mathrm{Cat}_{n}$ & 0 \\
$\mathrm{a}(G)$ & 1 & $\mathrm{Cat}_{n}$ & 0 \\
$\mathrm{~b}(G)$ & 1 & 0 & $(-1)^{n} \mathrm{Cat}_{n}$ \\
$\mathrm{c}_{2 i}(G)$ & $\delta_{i, 0}$ & $\mathrm{Cat}_{2 n-i, i}$ & $\mathrm{Cat}_{2 n+1-i, i}$ \\
\hline g.f. for $\mathrm{sa}(G ; t)$ & $\sum_{n \geq 0} \mathrm{sa}\left(P_{n} ; t\right) x^{n}=\frac{-1+2 t x+\sqrt{1+4 x^{2}}}{2 t x-2\left(t^{2}-1\right) x^{2}}$
\end{tabular}

Remark. For two given sequences $\sigma=\left(s_{0}, s_{1}, s_{2}, \ldots\right)$ and $\tau=\left(t_{1}, t_{2}, t_{3}, \ldots\right)$, define the generalized Catalan number $B_{n}$ by the sum of weighted Motzkin paths from $(0,0)$ to $(n, 0)$ with up steps $(1,1)$, horizontal steps $(1,0)$, and down steps $(1,-1)$ where we associate weight 1 to each up step, weight $s_{k}$ to each horizontal step on the line $y=k$, and weight $t_{k}$ to each down step between two lines $y=k-1$ and $y=k$. For example, if $\sigma \equiv 0$ and $\tau \equiv 1$, then $B_{2 n}=\mathrm{Cat}_{n}$. In Section 7.4 in [1], the generating function $B(z)=\sum_{n \geq 0} B_{n} z^{n}$ of the generalized Catalan number $B_{n}$ with $\sigma=(a, s, s, \ldots)$ and $\tau=(b, u, u, \ldots)$ is equal to

$$
B(z)=\frac{(2 u-b)+(b s-2 a u) z-b \sqrt{1-2 s x+\left(s^{2}-4 u\right) z^{2}}}{2(u-b)+2(b s-2 a u+a b) z+2\left(a^{2} u-a b s+b^{2}\right) z^{2}} .
$$

For $(a, s, b, u, z)=(t, 0,-1,-1, x)$, the formula (10) gives a combinatorial interpretation of the following formula

$$
\sum_{n \geq 0} \operatorname{sa}\left(P_{n} ; t\right) x^{n}=\frac{-1+2 t x+\sqrt{1+4 x^{2}}}{2 t x-2\left(t^{2}-1\right) x^{2}}
$$

and for $(a, s, b, u, z)=\left(0,0,-1, t^{2}-1, x\right)$, the formula (10) gives a combinatorial interpretation of the following formula

$$
\sum_{n \geq 0} \operatorname{sa}\left(P_{2 n} ; t\right) x^{2 n}=\frac{-\left(t^{2}+1\right)-\left(t^{2}-1\right) \sqrt{1+4 x^{2}}}{-2 t^{2}+2\left(t^{2}-1\right)^{2} x^{2}} .
$$

Theorem 2. Let $C_{n}$ be the cycle graph with $n$ vertices, which is a connected graph with all vertices of degree 2. Then the generating function for signed a-polynomials of $C_{n}$ is given by

$$
\sum_{n \geq 0} \operatorname{sa}\left(C_{n} ; t\right) x^{n}=\frac{1}{2}+\frac{1}{2 \sqrt{1+4 x^{2}}} \cdot \frac{\left(t^{2}+1\right) x+t \sqrt{1+4 x^{2}}}{t-\left(t^{2}-1\right) x} .
$$


TABLE 2. Numbers for cycle graphs $C_{n}$.

\begin{tabular}{c||c|c|c}
$G$ & $C_{0}$ & $C_{2 n}$ & $C_{2 n+1}$ \\
\hline $\mathrm{sa}(G)$ & 1 & $\frac{(-1)^{n}}{2}\left(\begin{array}{c}2 n \\
n\end{array}\right)$ & 0 \\
$\mathrm{a}(G)$ & 1 & $\frac{1}{2}\left(\begin{array}{c}2 n \\
n\end{array}\right)$ & 0 \\
$\mathrm{~b}(G)$ & 1 & 0 & $(-1)^{n}\left(\begin{array}{c}2 n \\
n\end{array}\right)$ \\
$\mathrm{c}_{2 i}(G)$ & $\delta_{i, 0}$ & $\left\{\begin{array}{c}\frac{1}{2}\left(\begin{array}{c}2 n \\
n\end{array}\right), \quad \text { if } i=n \\
\left(\begin{array}{c}2 n \\
i\end{array}\right),\end{array}\right.$ & if $i<n$
\end{tabular}

Proof. From Theorem 2.6 in [3], it is known that

$$
\mathrm{c}_{2 i}\left(C_{n}\right)= \begin{cases}1 & \text { if } i=n=0 \\
\frac{1}{2}\left(\begin{array}{c}
n \\
n / 2
\end{array}\right) & \text { if } 2 i=n>0 \\
\left(\begin{array}{c}
n \\
i
\end{array}\right) & \text { if } 2 i<n .\end{cases}
$$

Using formula (6), we obtain

$$
\begin{aligned}
\sum_{n \geq 0} \mathrm{sa}\left(C_{n} ; t\right) x^{n} & =\sum_{n \geq 0} \sum_{j=0}^{\lfloor n / 2\rfloor}(-1)^{j} \mathrm{c}_{2 j}\left(C_{n}\right) t^{n-2 j} x^{n} \\
& =\sum_{k \geq 0} \sum_{j \geq 0}(-1)^{j} \mathrm{c}_{2 j}\left(C_{2 j+k}\right) t^{k} x^{2 j+k} \\
& =\frac{1}{2}-\frac{1}{2} \sum_{j \geq 0}\left(\begin{array}{c}
2 j \\
j
\end{array}\right)\left(-x^{2}\right)^{j}+\sum_{k \geq 0} \sum_{j \geq 0}\left(\begin{array}{c}
2 j+k \\
j
\end{array}\right)(t x)^{k}\left(-x^{2}\right)^{j}
\end{aligned}
$$

From $\sum_{n \geq 0}\left(\begin{array}{c}2 n+k \\ n\end{array}\right) z^{n}=\frac{1}{1-\sqrt{1-4 z}}\left(\frac{1-\sqrt{1-4 z}}{2 z}\right)^{k}$, we have two generating functions:

$$
\begin{aligned}
\sum_{n \geq 0}\left(\begin{array}{c}
2 n \\
n
\end{array}\right) z^{n} & =\frac{1}{1-\sqrt{1-4 z}} \\
\sum_{n \geq 0} \sum_{k \geq 0}\left(\begin{array}{c}
2 n+k \\
n
\end{array}\right) w^{k} z^{n} & =\frac{1}{1-\sqrt{1-4 z}} \cdot \frac{1}{1-w\left(\frac{1-\sqrt{1-4 z}}{2 z}\right)} .
\end{aligned}
$$

Using the above two generating functions, (12) becomes (11). 
TABLE 3. Numbers for complete graphs $K_{n}$, where $\sum_{n \geq 0} A_{n} \frac{z^{n}}{n !}=\sec z+\tan z$.

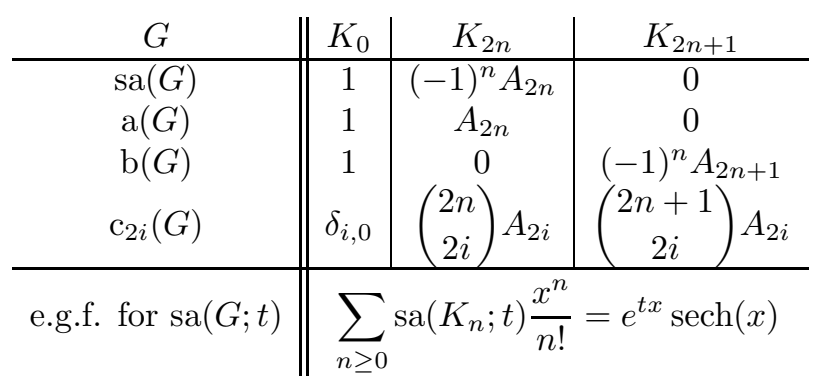

Let $A_{n}$ be the $n$-th Euler zigzag number for which the exponential generating function is

$$
\sum_{n \geq 0} A_{n} \frac{z^{n}}{n !}=\sec z+\tan z
$$

Theorem 3. Let $K_{n}$ be the complete graph with $n$ vertices. Then the exponential generating function for signed a-polynomials of $K_{n}$ is given by

$$
\sum_{n \geq 0} \operatorname{sa}\left(K_{n} ; t\right) \frac{x^{n}}{n !}=e^{t x} \operatorname{sech} x .
$$

Proof. From Theorem 2.8 in [3], it is known that

$$
\operatorname{sa}\left(K_{2 n}\right)=(-1)^{n} A_{2 n} .
$$

Using formula (5), we obtain

$$
\begin{aligned}
\sum_{n \geq 0} \operatorname{sa}\left(K_{n} ; t\right) \frac{x^{n}}{n !} & =\sum_{n \geq 0} \sum_{j=0}^{\lfloor n / 2\rfloor}\left(\begin{array}{c}
n \\
2 j
\end{array}\right) \operatorname{sa}\left(K_{2 j}\right) t^{n-2 j} \frac{x^{n}}{n !} \\
& =\sum_{k \geq 0} \sum_{j \geq 0}\left(\begin{array}{c}
k+2 j \\
2 j
\end{array}\right)(-1)^{j} A_{2 j} t^{k} \frac{x^{k+2 j}}{(k+2 j) !} \\
& =\left(\sum_{k \geq 0} \frac{(t x)^{k}}{k !}\right)\left(\sum_{j \geq 0} A_{2 j} \frac{(\imath x)^{2 j}}{(2 j) !}\right) .
\end{aligned}
$$

By (13), formula (15) becomes formula (14).

Remark. The Euler polynomials $E_{n}(t)$ is defined by the exponential generating function $\sum_{n \geq 0} E_{n}(t) \frac{x^{n}}{n !}=\left(\frac{2}{e^{x}+1}\right) e^{x t}$. See [4, p. 48]. Then it follows

$$
\mathrm{sa}\left(K_{n} ; t\right)=E_{n}\left(\frac{t+1}{2}\right) 2^{n}
$$


TABLE 4. Numbers for star graphs $K_{1, n-1}$, where $\sum_{n \geq 0} A_{n} \frac{z^{n}}{n !}=\sec z+\tan z$.

\begin{tabular}{|c|c|c|}
\hline$G$ & $K_{1,2 n-1}$ & $K_{1,2 n}$ \\
\hline $\mathrm{sa}(G)$ & $(-1)^{n} A_{2 n-1}$ & 0 \\
\hline $\mathrm{a}(G)$ & $A_{2 n-1}$ & 0 \\
\hline $\mathrm{b}(G)$ & 0 & $(-1)^{n} A_{2 n}$ \\
\hline $\mathrm{c}_{2 j}(G)$ & $\left\{\left(\begin{array}{c}2 n-1 \\
2 i-1\end{array}\right) A_{2 i-1}, \quad\right.$ if $i>0$ & $\int\left(\begin{array}{c}2 n \\
2 i-1\end{array}\right) A_{2 i-1}, \quad$ if $i>0$ \\
\hline & $\begin{cases}1, & \text { if } i=0\end{cases}$ & if $i=0$ \\
\hline e.g.f. for & \multicolumn{2}{|c|}{$\sum_{n \geq 0} \operatorname{sa}\left(K_{1, n} ; t\right) \frac{x^{n}}{n !}=e^{t x}(t-\tanh x)$} \\
\hline
\end{tabular}

from $\sum_{n \geq 0} \operatorname{sa}\left(K_{n} ; t\right) \frac{x^{n}}{n !}=e^{t x} \operatorname{sech} x=\left(\frac{2}{e^{2 x}+1}\right) e^{2 x\left(\frac{t+1}{2}\right)}=\sum_{n \geq 0} E_{n}\left(\frac{t+1}{2}\right) \frac{(2 x)^{n}}{n !}$.

Theorem 4. Let $K_{1, n}$ be the star graph with $n+1$ vertices, which is a tree with at least one vertex of degree $n$. Then the exponential generating function for signed a-polynomials of $K_{1, n}$ is given by

$$
\sum_{n \geq 0} \operatorname{sa}\left(K_{1, n} ; t\right) \frac{x^{n}}{n !}=e^{t x}(t-\tanh x) .
$$

Proof. From Theorem 2.9 in [3], it is known that

$$
\operatorname{sa}\left(K_{1,2 n+1}\right)=(-1)^{n+1} A_{2 n+1} .
$$

Using formula (5), we obtain

$$
\begin{aligned}
& \sum_{n \geq 0} \operatorname{sa}\left(K_{1, n} ; t\right) \frac{x^{n}}{n !} \\
= & \sum_{n \geq 0}\left(\operatorname{sa}(\emptyset) t^{n+1}+\sum_{j=0}^{\lfloor n / 2\rfloor}\left(\begin{array}{c}
n \\
2 j+1
\end{array}\right) \operatorname{sa}\left(K_{1,2 j+1}\right) t^{n-(2 j+1)}\right) \frac{x^{n}}{n !} \\
= & \sum_{n \geq 0} t^{n+1} \frac{x^{n}}{n !}+\sum_{k \geq 0} \sum_{j \geq 0}\left(\begin{array}{c}
k+2 j+1 \\
2 j+1
\end{array}\right)(-1)^{j+1} A_{2 j+1} t^{k} \frac{x^{k+2 j+1}}{(k+2 j+1) !} \\
(17)= & t\left(\sum_{n \geq 0} \frac{(t x)^{n}}{n !}\right)+\left(\sum_{k \geq 0} \frac{(t x)^{k}}{k !}\right)\left(\imath \sum_{j \geq 0} A_{2 j+1} \frac{(\imath x)^{2 j+1}}{(2 j+1) !}\right),
\end{aligned}
$$

where $\imath:=\sqrt{-1}$. By (13), it follows

$$
\sum_{j \geq 0} A_{2 j+1} \frac{(\imath x)^{2 j+1}}{(2 j+1) !}=\tan (\imath x)=\imath \tanh x
$$

and (17) becomes (16). 
Since $\operatorname{sa}(G)=\operatorname{sa}(G ; 0)$, putting $t=0$ in the generating functions (8), (11), (14), and (16) yields the generating functions for signed a-numbers of path graphs, cycle graphs, complete graphs, and star graphs as follows:

$$
\begin{aligned}
\sum_{n \geq 0} \mathrm{sa}\left(P_{n}\right) x^{n} & =\frac{-1+\sqrt{1+4 x^{2}}}{2 x^{2}}=\sum_{m \geq 0}(-1)^{m} \mathrm{Cat}_{m} x^{2 m}, \\
\sum_{n \geq 0} \mathrm{sa}\left(C_{n}\right) x^{n} & =\frac{1}{2}+\frac{1}{2 \sqrt{1+4 x^{2}}}=1+\sum_{m \geq 1} \frac{(-1)^{m}}{2}\left(\begin{array}{c}
2 m \\
m
\end{array}\right) x^{2 m}, \\
\sum_{n \geq 0} \operatorname{sa}\left(K_{n}\right) \frac{x^{n}}{n !} & =\operatorname{sech} x=\sum_{m \geq 0}(-1)^{m} A_{2 m} \frac{x^{2 m}}{(2 m) !} \\
\sum_{n \geq 0} \operatorname{sa}\left(K_{1, n}\right) \frac{x^{n}}{n !} & =-\tanh x=\sum_{m \geq 1}(-1)^{m} A_{2 m-1} \frac{x^{2 m-1}}{(2 m-1) !} .
\end{aligned}
$$

Similarly, since $\mathrm{b}(G)=\mathrm{sa}(G ; 1)$, putting $t=1$ in the generating functions (8), (11), (14), and (16) yields the generating functions for b-numbers of path graphs, cycle graphs, complete graphs, and star graphs as follows:

$$
\begin{aligned}
\sum_{n \geq 0} \mathrm{~b}\left(P_{n}\right) x^{n} & =1+\frac{-1+\sqrt{1+4 x^{2}}}{2 x}=1+\sum_{m \geq 0}(-1)^{m} \text { Cat }_{m} x^{2 m+1}, \\
\sum_{n \geq 0} \mathrm{~b}\left(C_{n}\right) x^{n} & =1+\frac{x}{\sqrt{1+4 x^{2}}}=1+\sum_{m \geq 0}(-1)^{m}\left(\begin{array}{c}
2 m \\
m
\end{array}\right) x^{2 m+1}, \\
\sum_{n \geq 0} \mathrm{~b}\left(K_{n}\right) \frac{x^{n}}{n !} & =1+\tanh x=1+\sum_{m \geq 0}(-1)^{m} A_{2 m+1} \frac{x^{2 m+1}}{(2 m+1) !} \\
\sum_{n \geq 0} \mathrm{~b}\left(K_{1, n}\right) \frac{x^{n}}{n !} & =\operatorname{sech} x=\sum_{m \geq 0}(-1)^{m} A_{2 m} \frac{x^{2 m}}{(2 m) !} .
\end{aligned}
$$

According to (7), putting $t \leftarrow \frac{1}{\sqrt{-z}}$ and $x \leftarrow x \sqrt{-z}$ in the generating functions (8), (11), (14), and (16) yields the next result.

Corollary 5. Let $P_{M(G)}(z)$ denote the Poincaré polynomials of the real toric manifolds $M(G)$ associated to the graph $G$. Then the generating functions for Poincaré polynomials of the real toric manifolds associated to path graphs $P_{n}$, cycle graphs $C_{n}$, complete graphs $K_{n}$, and star graphs $K_{1, n}$ are as follows:

$$
\begin{aligned}
& \sum_{n \geq 0} P_{M\left(P_{n}\right)}(z) x^{n}=\frac{-1+2 x+\sqrt{1-4 z x^{2}}}{2 x-2(1+z) x^{2}} \\
& \sum_{n \geq 0} P_{M\left(C_{n}\right)}(z) x^{n}=\frac{1}{2}+\frac{1}{2 \sqrt{1-4 z x^{2}}} \cdot \frac{(1-z) x+\sqrt{1-4 z x^{2}}}{1-(1+z) x}, \\
& \sum_{n \geq 0} P_{M\left(K_{n}\right)}(z) \frac{x^{n}}{n !}=e^{x} \sec (x \sqrt{z})
\end{aligned}
$$




$$
\sum_{n \geq 0} P_{M\left(K_{1, n}\right)}(z) \frac{x^{n}}{n !}=e^{x}(1+\sqrt{z} \tan (x \sqrt{z})) .
$$

\section{Signed a-number of complete multipartite graphs}

First, we consider the exponential generating function for signed a-numbers of complete bipartite graphs. Denote by $K_{p, q}$ the complete bipartite graph with $p$-set and $q$-set.

Theorem 6. The exponential generating function for signed a-numbers of complete bipartite graphs is

$$
\sum_{p \geq 0} \sum_{q \geq 0} \operatorname{sa}\left(K_{p, q}\right) \frac{x^{p}}{p !} \frac{y^{q}}{q !}=\frac{\cosh x+\cosh y-1}{\cosh (x+y)} .
$$

Proof. For two nonnegative integers $p$ and $q$ whose sum is even, there is the recurrence

$$
\sum_{i, j \geq 0}\left(\begin{array}{c}
p \\
i
\end{array}\right)\left(\begin{array}{l}
q \\
j
\end{array}\right) \mathrm{sa}\left(K_{i, j}\right)= \begin{cases}0 & \text { if } p \text { and } q \text { are positive } \\
1 & \text { if } p \text { or } q \text { is zero. }\end{cases}
$$

The exponential generating function for the right-hand side of (19) is

$$
\sum_{\substack{p, q \geq 0 \\ p+q=\text { even }}}(R H S) \frac{x^{p}}{p !} \frac{y^{q}}{q !}=1+(\cosh x-1)+(\cosh y-1) .
$$

The exponential generating function for the left-hand side of (19) is

$$
\begin{aligned}
\sum_{\substack{p, q \geq 0 \\
p+q=\text { even }}}(L H S) \frac{x^{p}}{p !} \frac{y^{q}}{q !} & =\sum_{\substack{p, q \geq 0 \\
p+q=\text { even }}} \sum_{\substack{0 \leq i \leq p \\
0 \leq j \leq \leq \\
i+j=\text { even }}}\left(\operatorname{sa}\left(K_{i, j}\right) \frac{x^{i}}{i !} \frac{y^{j}}{j !}\right)\left(\frac{x^{p-i}}{(p-i) !} \frac{y^{q-j}}{(q-j) !}\right) \\
& =\left(\sum_{\substack{i, j \geq 0 \\
i+j=\mathrm{even}}} \operatorname{sa}\left(K_{i, j}\right) \frac{x^{i}}{i !} \frac{y^{j}}{j !}\right)\left(\sum_{\substack{i, j \geq 0 \\
i+j=\mathrm{even}}} \frac{x^{i}}{i !} \frac{y^{j}}{j !}\right) \\
& =\left(\sum_{\substack{p, q \geq 0 \\
21)}} \operatorname{sa}\left(K_{p, q}\right) \frac{x^{p}}{p !} \frac{y^{q}}{q !}\right) \cosh (x+y) .
\end{aligned}
$$

Thus, by (20) and (21), we are done.

The generating function $S A_{q}(x)$ is defined by $S A_{q}(x)=\sum_{p \geq 0} \operatorname{sa}\left(K_{p, q}\right) \frac{x^{p}}{p !}$, which is the coefficient of $y^{q} / q$ ! in $\frac{\cosh x+\cosh y-1}{\cosh (x+y)}$. Given a fixed nonnegative $q$, we can induce the detailed formula $S A_{q}(x)$ by

$$
S A_{q}(x)=\left.\frac{\partial^{q}}{\partial y^{q}}\left(\frac{\cosh x+\cosh y-1}{\cosh (x+y)}\right)\right|_{y=0} .
$$


For example, the initial generating functions $A_{q}(x)$ are listed as follows:

$$
\begin{aligned}
& S A_{0}(x)=1 \\
& S A_{1}(x)=-\tanh x \\
& S A_{2}(x)=-2 \operatorname{sech}^{2} x+\operatorname{sech} x+1 \\
& S A_{3}(x)=\left(6 \operatorname{sech}^{2} x-3 \operatorname{sech} x-1\right) \tanh x \\
& S A_{4}(x)=24 \operatorname{sech}^{4} x-12 \operatorname{sech}^{3} x-20 \operatorname{sech}^{2} x+7 \operatorname{sech} x+1 .
\end{aligned}
$$

Next, we generalize the generating function (18) for complete multipartite graphs. Denote by $K_{p_{1}, \ldots, p_{m}}$ the complete $m$-partite graph with $p_{1}$-set, ..., $p_{m}$-set.

Theorem 7. The exponential generating function for signed a-numbers of complete $m$-partite graphs is

$$
\sum_{p_{1}, \ldots, p_{m} \geq 0} \operatorname{sa}\left(K_{p_{1}, \ldots, p_{m}}\right) \frac{x_{1}^{p_{1}}}{p_{1} !} \cdots \frac{x_{m}^{p_{m}}}{p_{m} !}=\frac{(1-m)+\cosh x_{1}+\cdots+\cosh x_{m}}{\cosh \left(x_{1}+\cdots+x_{m}\right)} .
$$

Proof. For $m$ nonnegative integers $p_{1}, \ldots, p_{m}$ whose sum is even, there is the recurrence

$$
\begin{gathered}
\sum_{i_{1}, \ldots, i_{m} \geq 0}\left(\begin{array}{c}
p_{1} \\
i_{1}
\end{array}\right) \ldots\left(\begin{array}{c}
p_{m} \\
i_{m}
\end{array}\right) \operatorname{sa}\left(K_{i_{1}, \ldots, i_{m}}\right) \\
= \begin{cases}0 & \text { if at least two } p_{i} \text { 's are positive, } \\
1 & \text { if all } p_{i} \text { 's are zeros, but at most one. }\end{cases}
\end{gathered}
$$

Using both sides of (23), we have the generalized formulas of (20) and (21) as follows:

$$
\sum_{\substack{p_{i} \geq 0 \\ p_{1}+\cdots+p_{m}=\text { even }}}(R H S) \frac{x_{1}^{p_{1}}}{p_{1} !} \ldots \frac{x_{m}^{p_{m}}}{p_{m} !}=1+\left(\cosh x_{1}-1\right)+\cdots+\left(\cosh x_{m}-1\right)
$$

and

$$
\begin{gathered}
\sum_{\substack{p_{i} \geq 0 \\
p_{1}+\cdots+p_{m}=\text { even }}}(\text { LHS }) \frac{x_{1}^{p_{1}}}{p_{1} !} \ldots \frac{x_{m}^{p_{m}}}{p_{m} !} \\
=\left(\sum_{p_{1}, \ldots, p_{m} \geq 0} \operatorname{sa}\left(K_{p_{1}, \ldots, p_{m}}\right) \frac{x_{1}^{p_{1}}}{p_{1} !} \cdots \frac{x_{m}^{p_{m}}}{p_{m} !}\right) \cosh \left(x_{1}+\cdots+x_{m}\right),
\end{gathered}
$$

which completes the proof.

Remark. Since a $\left(K_{p_{1}, \ldots, p_{m}}\right)=(-1)^{\frac{p_{1}+\cdots+p_{m}}{2}} \operatorname{sa}\left(K_{p_{1}, \ldots, p_{m}}\right)$ and $\cosh (\imath z)=\cos z$, the exponential generating functions for a-numbers of complete bipartite graphs 
and complete $m$-partite graphs are equal to

$$
\begin{gathered}
\sum_{p \geq 0} \sum_{q \geq 0} \mathrm{a}\left(K_{p, q}\right) \frac{x^{p}}{p !} \frac{y^{q}}{q !}=\frac{\cos x+\cos y-1}{\cos (x+y)}, \\
\sum_{p_{1}, \ldots, p_{m} \geq 0} \mathrm{a}\left(K_{p_{1}, \ldots, p_{m}}\right) \frac{x_{1}^{p_{1}}}{p_{1} !} \cdots \frac{x_{m}^{p_{m}}}{p_{m} !}=\frac{(1-m)+\cos x_{1}+\cdots+\cos x_{m}}{\cos \left(x_{1}+\cdots+x_{m}\right)} .
\end{gathered}
$$

\section{Signed a-polynomial of complete multipartite graphs}

First, we consider the exponential generating function for signed a-polynomials of complete bipartite graphs.

Theorem 8. Let $K_{p, q}$ be the complete bipartite graph with p-set and q-set. Then the exponential generating function for signed a-polynomials of $K_{p, q}$ is given by

$$
\sum_{p \geq 0} \sum_{q \geq 0} \operatorname{sa}\left(K_{p, q} ; t\right) \frac{x^{p}}{p !} \frac{y^{q}}{q !}=e^{t(x+y)}\left(\frac{\cosh x+\cosh y-1}{\cosh (x+y)}\right) .
$$

Proof. By definition, we have

$$
\sum_{\substack{p \geq 0 \\
q \geq 0}} \operatorname{sa}\left(K_{p, q} ; t\right) \frac{x^{p}}{p !} \frac{y^{q}}{q !}=\sum_{\substack{p \geq 0 \\
q \geq 0}}\left(\sum_{\substack{0 \leq p^{\prime} \leq p \\
0 \leq q^{\prime} \leq q}}\left(\begin{array}{c}
p \\
p^{\prime}
\end{array}\right)\left(\begin{array}{c}
q \\
q^{\prime}
\end{array}\right) \operatorname{sa}\left(K_{p^{\prime}, q^{\prime}}\right) t^{p-p^{\prime}+q-q^{\prime}}\right) \frac{x^{p}}{p !} \frac{y^{q}}{q !} .
$$

Substituting $p^{\prime \prime}=p-p^{\prime}$ and $q^{\prime \prime}=q-q^{\prime}$, the right-hand side of (25) becomes

$$
\begin{aligned}
& \sum_{\substack{p^{\prime \prime} \geq 0 \\
q^{\prime \prime} \geq 0}}\left(\sum_{\substack{p^{\prime} \geq 0 \\
q^{\prime} \geq 0}}\left(\begin{array}{c}
p^{\prime}+p^{\prime \prime} \\
p^{\prime}
\end{array}\right)\left(\begin{array}{c}
q^{\prime}+q^{\prime \prime} \\
q^{\prime}
\end{array}\right) \operatorname{sa}\left(K_{p^{\prime}, q^{\prime}}\right) t^{p^{\prime \prime}+q^{\prime \prime}}\right) \frac{x^{p^{\prime}+p^{\prime \prime}}}{\left(p^{\prime}+p^{\prime \prime}\right) !} \frac{y^{q^{\prime}+q^{\prime \prime}}}{\left(q^{\prime}+q^{\prime \prime}\right) !} \\
= & \left(\sum_{p^{\prime} \geq 0} \sum_{q^{\prime} \geq 0} \operatorname{sa}\left(K_{p^{\prime}, q^{\prime}}\right) \frac{x^{p^{\prime}}}{p^{\prime} !} \frac{y^{q^{\prime}}}{q^{\prime} !}\right)\left(\sum_{p^{\prime \prime} \geq 0} \frac{(t x)^{p^{\prime \prime}}}{p^{\prime \prime} !}\right)\left(\sum_{q^{\prime \prime} \geq 0} \frac{(t y)^{q^{\prime \prime}}}{q^{\prime \prime !}}\right) .
\end{aligned}
$$

The formula (18) completes the proof.

Remark. Since the coefficient of $\frac{y^{q}}{q !}$ in formula (24) is equal to

$$
\sum_{n \geq 0} \operatorname{sa}\left(K_{q, n} ; t\right) \frac{x^{n}}{n !}
$$

it holds that

$$
\sum_{n \geq 0} \operatorname{sa}\left(K_{q, n} ; t\right) \frac{x^{n}}{n !}=\left.\frac{\partial^{q}}{\partial y^{q}} e^{t(x+y)}\left(\frac{\cosh x+\cosh y-1}{\cosh (x+y)}\right)\right|_{y=0} .
$$

In case of $q=1$, we have the exponential generating function (16) for signed a-polynomials of star graphs again. 
Similarly, we can deduce the next theorem by the same above method.

Theorem 9. Let $K_{p_{1}, \ldots, p_{m}}$ be the complete $m$-partite graph with $p_{1}$-set, ..., $p_{m}$-set. Then the exponential generating function for signed a-polynomials of $K_{p_{1}, \ldots, p_{m}}$ is given by

$$
\begin{gathered}
\sum_{p_{1} \ldots, p_{m} \geq 0} \operatorname{sa}\left(K_{p_{1}, \ldots, p_{m}} ; t\right) \frac{x_{1}^{p_{1}}}{p_{1} !} \ldots \frac{x_{m}^{p_{m}}}{p_{m} !} \\
=e^{t\left(x_{1}+\cdots+x_{m}\right)}\left(\frac{(1-m)+\cosh x_{1}+\cdots+\cosh x_{m}}{\cosh \left(x_{1}+\cdots+x_{m}\right)}\right) .
\end{gathered}
$$

Since $\operatorname{sa}(G)=\operatorname{sa}(G ; 0)$, putting $t=0$ in the generating functions (24) and (26) gives the two formulas (18) and (22), respectively. Also, since $\mathrm{b}(G)=$ $\mathrm{sa}(G ; 1)$, putting $t=1$ in the generating functions (24) and (26) yields the generating functions for b-numbers of complete bipartite graphs and complete multipartite graphs as follows:

$$
\begin{gathered}
\sum_{p \geq 0} \sum_{q \geq 0} \mathrm{~b}\left(K_{p, q}\right) \frac{x^{p}}{p !} \frac{y^{q}}{q !}=e^{x+y}\left(\frac{\cosh x+\cosh y-1}{\cosh (x+y)}\right), \\
\sum_{p_{1} \ldots, p_{m} \geq 0} \mathrm{~b}\left(K_{p_{1}, \ldots, p_{m}}\right) \frac{x_{1}^{p_{1}}}{p_{1} !} \cdots \frac{x_{m}^{p_{m}}}{p_{m} !} \\
=e^{x_{1}+\cdots+x_{m}}\left(\frac{(1-m)+\cosh x_{1}+\cdots+\cosh x_{m}}{\cosh \left(x_{1}+\cdots+x_{m}\right)}\right) .
\end{gathered}
$$

The next result follows from two generating functions (24) and (26) by plugging in (7).

Corollary 10. Let $P_{M\left(K_{p, q}\right)}(z)$ and $P_{M\left(K_{\left.p_{1}, \ldots, p_{m}\right)}\right.}(z)$ denote the Poincaré polynomials of the real toric manifolds associated to the complete bipartite graph $K_{p, q}$ and the complete $m$-partite graph $K_{p_{1}, \ldots, p_{m}}$. Then the generating functions for Poincaré polynomials $P_{M\left(K_{p, q}\right)}(z)$ and $P_{M\left(K_{p_{1}, \ldots, p_{m}}\right)}(z)$ are equal to

$$
\begin{aligned}
\sum_{n \geq 0} P_{M\left(K_{p, q}\right)}(z) \frac{x^{p}}{p !} \frac{y^{q}}{q !}=e^{x+y}\left(\frac{\cos (x \sqrt{z})+\cos (y \sqrt{z})-1}{\cos (x \sqrt{z}+y \sqrt{z})}\right), \\
\quad \sum_{n \geq 0} P_{M\left(K_{p_{1}, \ldots, p_{m}}\right)}(z) \frac{x_{1}^{p_{1}}}{p_{1} !} \ldots \frac{x_{m}^{p_{m}}}{p_{m} !} \\
=e^{x_{1}+\cdots+x_{m}}\left(\frac{(1-m)+\cos \left(x_{1} \sqrt{z}\right)+\cdots+\cos \left(x_{m} \sqrt{z}\right)}{\cos \left(x_{1} \sqrt{z}+\cdots+x_{m} \sqrt{z}\right)}\right) .
\end{aligned}
$$

Table 5 shows the Poincaré polynomials $P_{M\left(K_{p, q}\right)}(z)$ for $p \leq 6$ and $q \leq 3$. 
TABLE 5. Table for $P_{M\left(K_{p, q}\right)}(z)$

\begin{tabular}{c|llll}
$p \backslash q$ & 0 & 1 & 2 & 3 \\
\hline 0 & 1 & 1 & 1 & 1 \\
1 & 1 & $1+z$ & $1+2 z$ & $1+3 z+2 z^{2}$ \\
2 & 1 & $1+2 z$ & $1+4 z+3 z^{2}$ & $1+6 z+13 z^{2}$ \\
3 & 1 & $1+3 z+2 z^{2}$ & $1+6 z+13 z^{2}$ & $1+9 z+39 z^{2}+31 z^{3}$ \\
4 & 1 & $1+4 z+8 z^{2}$ & $1+8 z+34 z^{2}+27 z^{3}$ & $1+12 z+86 z^{2}+205 z^{3}$ \\
5 & 1 & $1+5 z+20 z^{2}+16 z^{3}$ & $1+10 z+70 z^{2}+167 z^{3}$ & $1+15 z+160 z^{2}+763 z^{3}+617 z^{4}$ \\
6 & 1 & $1+6 z+40 z^{2}+96 z^{3}$ & $1+12 z+125 z^{2}+597 z^{3}+483 z^{4}$ & $1+18 z+267 z^{2}+2123 z^{3}+5151 z^{4}$
\end{tabular}

\section{Remarks}

We have found the signed a-polynomial of complete multipartite graph. For a general graph $G$, it is not easy to characterize sa $(G ; t)$. Even the case of the tree, it is hard to find the close formula of its signed a-numbers. For example, letting $T_{p, q, r}$ be the tree induced by connecting one vertex and each end-vertices of $P_{p}, P_{q}$, and $P_{r}$ by three edges, we are not able to find a closed form of $\mathrm{sa}\left(T_{p, q, r} ; t\right)$ with $p, q$, and $r$. In order to find the general formulas for the signed a-polynomial of any graph, we need a method to calculate the signed a-numbers of a specific graph without a recursive definition.

Acknowledgment. For to the first author, this research was supported by Basic Science Research Program through the National Research Foundation of Korea (NRF) funded by the Ministry of Education. For the second author, this research was supported by Basic Science Research Program through the National Research Foundation of Korea (NRF) funded by the Ministry of Science, ICT \& Future Planning and Inha University Research Grant (INHA-44756).

\section{References}

[1] M. Aigner, A Course in Enumeration, Graduate Texts in Mathematics, vol. 238, Springer, Berlin, 2007.

[2] M. P. Carr and S. L. Devadoss, Coxeter complexes and graph-associahedra, Topology Appl. 153 (2006), no. 12, 2155-2168.

[3] S. Choi and H. Park, A new graph invariant arises in toric topology, accepted in J. Math. Soc. Japan (2014), available at arXiv:1210.3776.

[4] L. Comtet, Advanced Combinatorics, enlarged ed., D. Reidel Publishing Co., Dordrecht, 1974, The art of finite and infinite expansions.

[5] A. Postnikov, Permutohedra, associahedra, and beyond, Int. Math. Res. Not. IMRN (2009), no. 6, 1026-1106.

[6] A. Postnikov, V. Reiner, and L. Williams, Faces of generalized permutohedra, Doc. Math. 13 (2008), 207-273.

SEunghyun SEO

Department of Mathematics Education

KANGWON NATIONAL UNIVERSITY

Chuncheon 200-701, KoreA

E-mail address: shyunseo@kangwon.ac.kr 
HeEsung Shin

Department of Mathematics

INHA UNIVERSITY

INCHEON 402-751, KOREA

E-mail address: shin@inha.ac.kr 